Pesq. Vet. Bras. 37(5):432-440, maio 2017

DOI: $10.1590 / \mathrm{S} 0100-736 \mathrm{X} 2017000500002$

\title{
Doenças de bovinos em Mato Grosso diagnosticadas no Laboratório de Patologia Veterinária da UFMT (2005-2014) ${ }^{1}$
}

\begin{abstract}
Leilane A.S. Rondelli ${ }^{2}$, Gustavo S. Silva ${ }^{3}$, Kalinne S. Bezerra ${ }^{2}$, André L.H. Rondelli², Samara R. Lima ${ }^{2}$, Fernando H. Furlan ${ }^{4}$, Caroline A. Pescador ${ }^{5}$ e Edson M. Colodel ${ }^{5}$

ABSTRACT.- Rondelli L.A.S., Silva G.S., Bezerra K.S., Rondelli A.L.H., Lima S.R., Furlan F.H., Pescador C.A. \& Colodel E.M. 2017. [Cattle diseases in Mato Grosso diagnosed in the Veterinary Pathology Laboratory of UFMT (2005-2014).] Doenças de bovinos no Estado de Mato Grosso diagnosticadas no Laboratório de Patologia Veterinária da UFMT (20052014). Pesquisa Veterinária Brasileira 37(5):432-440. Departamento de Clinica Médica Veterinária, Faculdade de Agronomia, Medicina Veterinária e Zootecnia, Universidade Federal de Mato Grosso, Av. Fernando Corrêa da Costa 2367, Bairro Boa Esperança, Cuiabá, MT 78060-900, Brazil. E-mail: leilane_go@hotmail.com

A survey was conducted on files of the Veterinary Pathology Laboratory (LPV), Federal University of Mato Grosso (UFMT), about cattle disease recorded during the years 2005 to 2014. From a total of 1124 reviewed cases, $27.6 \%$ were samples obtained from autopsies performed by technicians of the LPV-UFMT, and $72.3 \%$ were samples sent to LPV-UFMT by field veterinarians. In $49.38 \%$ of cases $(555 / 1124)$ the diagnosis of the disease was made by morphological analysis of lesions and/or through additional tests. Rabies was the major cause of death in cattle in this study (7.82\%). Inflammatory and parasitic disease was the most prevalent category diagnosed with $27.49 \%$ of cases, followed by toxicoses and toxinfections (9.78\%). The other categories were distributed in descending order, as neoplasms and tumor-like lesions (4\%), degenerative diseases (3.02\%), disorders caused by physical agents $(2.84 \%)$, metabolic and nutritional disorders (1.42\%), and other categories $(0.71 \%)$.
\end{abstract}

INDEX TERMS: Cattle diseases, Mato Grosso, pathology, retrospective study.

RESUMO.- Foi realizado um levantamento nos arquivos do Laboratório de Patologia Veterinária (LPV) da Universidade Federal de Mato Grosso (UFMT) das doenças de bovinos registradas entre os anos 2005 a 2014. Foram revisados 1124 casos. Destes, 27,6\% foram amostras obtidas de necropsias realizadas por técnicos do LPV-UFMT

\footnotetext{
${ }^{1}$ Recebido em 21 de agosto de 2015.

Aceito para publicação em 30 de agosto de 2016.

${ }^{2}$ Laboratório de Patologia Veterinária, Hospital Veterinário, Universidade Federal de Mato Grosso (UFMT), Av. Fernando Corrêa da Costa 2367, Boa Esperança, Cuiabá, MT 78060-900, Brasil. *Autor para correspondência: leilane_go@hotmail.com

${ }^{3}$ Laboratório de Epidemiologia Veterinária (EPILAB), Universidade Federal do Rio Grande do Sul, Av. Bento Gonçalves 9090, Agronomia, Porto Alegre, RS 90540-900, Brasil.

${ }^{4}$ Departamento de Ciências Básicas e Produção Animal, Faculdade de Agronomia, Medicina Veterinária e Zootecnia, UFMT, Av. Fernando Corrêa da Costa 2367, Bairro Boa Esperança, Cuiabá, MT 78060-900, Brasil.

${ }^{5}$ Departamento de Clínica Médica Veterinária, Faculdade de Agronomia, Medicina Veterinária e Zootecnia, UFMT, Av. Fernando Corrêa da Costa 2367, Bairro Boa Esperança, Cuiabá, MT 78060-900, Brasil.
}

e 72,3\% foram amostras encaminhadas ao LPV-UFMT por veterinários de campo. Em 49,38\% dos casos (555/1124) o diagnóstico da doença foi feito através da análise morfológica de lesões e/ou através de exames complementares. Raiva foi a principal causa de morte de bovinos neste estudo $(7,82 \%)$. As doenças inflamatórias e parasitárias foram as mais prevalentes sendo diagnosticadas em $27,49 \%$ dos casos, seguida das doenças tóxicas e toxiinfecções com $9,78 \%$. As demais categorias foram distribuídas em ordem decrescente em: neoplasmas e lesões tumoriformes (4\%), doenças degenerativas $(3,02 \%)$, distúrbios causados por agentes físicos $(2,84 \%)$, distúrbios metabólicos e nutricionais $(1,42 \%)$ e outras categorias $(0,71 \%)$.

TERMOS DE INDEXAÇÃO: Doenças de bovinos, Mato Grosso, patologia, estudo retrospectivo.

\section{INTRODUÇÃO}

No Brasil a atividade agrária é responsável por uma importante parcela do Produto Interno Bruto. A pecuária bovina é um dos setores mais importantes do agronegócio brasi- 
leiro e consequentemente da economia nacional. O Brasil possui o maior rebanho comercial do mundo, é o maior exportador de carne bovina, segundo maior produtor de carne e sexto maior produtor de leite (USDA 2014). Em 2013 o país produziu 9,6 milhões de toneladas de carne, dos quais 7,6 milhões foram destinadas ao mercado interno (Conab 2014). Além disso, as exportações de carne crescem a cada ano para um número cada vez maior de mercados devido às conquistas obtidas com a defesa agropecuária. Nesse contexto, o Estado de Mato Grosso possui o maior rebanho bovino do Brasil, com 28.487,977 milhões de bovinos distribuídos em 102.162 mil propriedades rurais (INDEA 2014). 0 faturamento gerado em 2014 pela exportação de carne bovina produzida em Mato Grosso foi de $\mathrm{R} \$ 1,2$ bilhão, que representa $18,71 \%$ de toda a exportação da carne bovina brasileira (ACRIMAT 2015), todavia, apesar da destacada expansão deste produto, principalmente na região Centro-Oeste (IBGE 2012), sabe-se da necessidade e das possibilidades de incrementar a produtividade da bovinocultura do Estado. A eficiência da produtividade na bovinocultura está diretamente relacionada à sanidade do rebanho, uma vez que um rebanho doente tem sua produtividade comprometida pela menor eficiência ou até mesmo a morte dos animais. No Brasil, pelo menos 5\% do rebanho bovino morre anualmente por diferentes causas (Pessoa et al. 2013).

Um estudo retrospectivo realizado na região Sul do Brasil, apontou que as doenças tóxicas e toxiinfecções, agregado a doenças inflamatórias e parasitárias são responsáveis por $77,2 \%$ dessas mortes (Lucena et al. 2010). 0 conhecimento sobre a prevalência das diferentes doenças que afetam bovinos de uma região é fundamental para que médicos veterinários que trabalham a campo possam usufruir de uma lista de prováveis causas de mortalidade em bovinos na região embasando o diagnóstico diferencial e facilitando a coleta correta de amostras e seu envio para exames complementares (Lucena et al. 2010). No estado de Mato Grosso, embora muitos estudos tenham sido conduzidos isoladamente para importantes doenças que afetam a produção de bovinos (Mendonça et al. 2008, Arruda et al. 2010, Ubiali et al. 2011, Gaeti et al. 2014), não existe uma compilação de dados sobre as principais enfermidades que acometem os rebanhos desta região (Ubiali et al. 2008). Objetiva-se determinar a frequência e características clínicas, patológicas e epidemiológicas das doenças de bovinos no Estado do Mato Grosso através do estudo retrospectivo de diagnósticos morfológicos registrados no Laboratório de Patologia Veterinária (LPV) da Universidade Federal de Mato Grosso (UFMT) entre os anos de 2005 a 2014.

\section{MATERIAL E MÉTODOS}

Foram revisados os arquivos de laudos do Laboratório de Patologia Veterinária (LPV) da Universidade Federal de Mato Grosso (UFMT) referentes a necropsias realizadas, em bovinos, pela equipe do LPV-UFMT ou encaminhadas por veterinários, autônomos ou vinculados a empresas, de diferentes municípios do Estado de Mato Grosso entre janeiro de 2005 e dezembro de 2014. Não foram computados dados de bovinos utilizados em experimentos ou fetos bovinos abortados. Informações epidemiológicas referentes à idade, raça, sexo, data de envio da amostra, localização, sinais clínicos, alterações em exames laboratoriais, lesões macroscópicas e histológicas e diagnóstico definitivo foram verificadas nos livros de registro e computados em uma planilha. Os diagnósticos foram classificados em conclusivos e inconclusivos. Este último foi assim classificado quando as alterações morfológicas não eram consistentes para caracterização da causa do quadro clínico. Nestes casos os achados clínico-laboratoriais e as lesões encontradas na necropsia foram reavaliados, nos laudos, a fim de se tentar estabelecer um diagnóstico de acordo com a literatura atual. Quando as evidências não permitiram estabelecer um diagnóstico definitivo, os casos foram considerados inconclusivos.

Os casos conclusivos foram classificados de acordo com critérios adotados por Lucena et al. (2010). Cada diagnóstico atribuído referiu-se à ocorrência da doença em uma propriedade, podendo tratar-se de um único bovino ou um surto. Para a avaliação estatística foi realizada uma análise descritiva do banco de dados sendo posteriormente averiguada a distribuição de frequências dos casos recebidos e suas classificações.

\section{RESULTADOS}

A espécie bovina representou 8,51\% (1124/13.199) de todos os exames realizados no LPV-UFMT. De janeiro de 2005 a dezembro de 2014 foram examinados, 311 casos de doenças de bovinos submetidos a necropsia pela equipe do LPV-UFMT e 813 amostras encaminhadas para o LPV-UFMT perfazendo um total de 1.124 casos. As amostras encaminhadas foram provenientes das cinco mesorregiões do estado, sendo $434(38,61 \%)$ da região Centro-Sul, 256 $(22,78 \%)$ da região Norte, $143(12,72 \%)$ da região Sudoeste, $111(9,87 \%)$ da região Sudeste e $5(4,90 \%)$ da região nordeste. Em $125(11,12 \%)$ casos a localização não foi informada. Dos 1.124 casos, 537 (47,78\%) eram fêmeas, 373 $(33,19 \%)$ eram machos e em $214(19,03 \%)$ o sexo não foi informado. Um total de 15 raças de bovinos foram afetadas, dentre elas a Nelore com 566 (50,36\%), Mestiço (SRD) com 151 (13,43\%), Girolando com 34 (3,02\%), Holandesa com 18 (1,60\%), Gir com 17 (1,51\%), Caracu com 7 (0,62\%), Brahman e Brangus com $5(0,44 \%)$ cada, Simental com 4 $(0,36 \%)$ Blonde d'aquitaine com 3(0,27\%), Pardo Suíço e Senepol com $2(0,18 \%)$ cada e Canchim, Jersey e Rubia Galega com $1(0,09 \%)$ cada. Os principais dados referentes a origem e raça dos bovinos analisados no LPV-UFMT entre os anos de 2005 a 2014 estão sumarizados no Quadro 1. Dos 1124 casos avaliados neste estudo, $554(49,28 \%)$ tinham diagnóstico conclusivo e em 570 casos $(50,71 \%)$ não foi possível estabelecer um diagnóstico definitivo. Dentre os casos em que o diagnóstico foi considerado inconclusivo, $98(17,19 \%)$ eram exames provenientes de necropsias realizadas pela equipe do LPV-UFMT e 472 casos $(82,80 \%)$ tratavam-se de amostras encaminhadas ao LPV-UFMT.

Considerando os 554 casos em que o diagnóstico foi classificado como conclusivo, as doenças inflamatórias e parasitárias foram as mais prevalentes, diagnosticadas em 309 (55,77\%) casos, seguidas das doenças tóxicas e/ ou toxiinfecções que acometeram 110 (19,85\%) bovinos. Os demais diagnósticos conclusivos foram distribuídos em ordem decrescente e classificados em: neoplasmas e lesões tumoriformes em (44 casos/7,94\%), doenças degenerativas (34 casos/6,13\%), distúrbios causados por agentes físicos (32 casos/5,77\%), distúrbios metabólicos e nutricio- 
Quadro 1. Dados sobre origem, sexo, raça e aptidão de bovinos analisados no LPV-UFMT nos anos de 2005 a 2014

\begin{tabular}{|c|c|c|c|}
\hline \multirow{2}{*}{$\begin{array}{l}\text { Epidemiologia geral } \\
\text { Mesorregião de MT/no de cidades }\end{array}$} & \multicolumn{3}{|c|}{ Categoria } \\
\hline & $\mathrm{H}$ & $\mathrm{N}$ & Tota \\
\hline Centro-Sul/19 & 246 & 188 & 434 \\
\hline Norte/43 & 212 & 44 & 256 \\
\hline Sudoeste/22 & 127 & 16 & 143 \\
\hline Sudeste/16 & 84 & 27 & 111 \\
\hline Nordeste/12 & 44 & 11 & 55 \\
\hline $\mathrm{NI} / 125$ & 100 & 25 & 125 \\
\hline \multicolumn{4}{|l|}{ Sexo } \\
\hline Fêmeas & 366 & 171 & 537 \\
\hline Machos & 259 & 114 & 373 \\
\hline $\mathrm{NI}$ & 183 & 31 & 214 \\
\hline \multicolumn{4}{|l|}{ Raças } \\
\hline Nelore & 402 & 164 & 566 \\
\hline Mestiço & 96 & 55 & 151 \\
\hline Girolando & 12 & 22 & 34 \\
\hline Holandesa & 12 & 6 & 18 \\
\hline Gir & 5 & 12 & 17 \\
\hline Caracu & 4 & 3 & 7 \\
\hline Brahman & 1 & 4 & 5 \\
\hline Brangus & 2 & 3 & 5 \\
\hline Simental & 3 & 1 & 4 \\
\hline Blonde D'aquitaine & 2 & 1 & 3 \\
\hline Pardo-Suíço & 2 & 0 & 2 \\
\hline Senepol & 2 & 0 & 2 \\
\hline Jersey & 0 & 1 & 1 \\
\hline Canchim & 1 & 0 & 1 \\
\hline Rubia Gallega & 1 & 0 & 1 \\
\hline NI & 268 & 39 & 307 \\
\hline \multicolumn{4}{|l|}{ Aptidão } \\
\hline Corte & 510 & 230 & 740 \\
\hline Leite & 35 & 42 & 77 \\
\hline
\end{tabular}

$\mathrm{NI}=$ não informado, $\mathrm{H}=$ histológico, $\mathrm{N}=$ necropsia

nais (16 casos/2,88\%), outras categorias (8 casos/1,44\%). A relação da classificação das doenças e a distribuição da idade dos animais acometidos encontra-se representada no Quadro 2. As principais doenças categorizadas de acordo com o grupo de origem estão sumarizadas nos Quadros 3,4 e 5 .

\section{DISCUSSÃO}

Propriedades com grande dimensão territorial com utilização de bovinos de raças azebuadas, principalmente nelore, mantidos em sistema extensivo de criação são características predominantes na bovinocultura do estado de Mato Grosso (ACRIMAT 2012). Essa peculiaridade justifica a divergência dos resultados observados neste estudo em relação a outros levantamentos sobre causas de morte de bo- vinos realizados em regiões onde existe a predominância do sistema intensivo. Considerando o grupo das doenças inflamatórias e parasitárias, as enfermidades que afetam exclusivamente o sistema respiratório foram responsáveis por $7,77 \%$ das causas de mortalidade de bovinos (24/309). Estudos mostram que em confinamentos as doenças respiratórias são responsáveis por $54 \%$ a $66 \%$ das causas de mortalidade de bovinos (Martin et al. 1980, Martin et al. 1981, Gagea et al. 2006). Os estados de Goiás, São Paulo e Mato Grosso são os maiores confinadores atualmente (IBGE 2012). Apesar do menor crescimento de confinamentos em 2014, os produtores em maioria se mostram confiantes em aumentar a quantidade de bovinos confinados para os anos seguintes (IMEA 2014), fato que poderá aumentar a importância das doenças respiratórias como causa de perda produtiva para a bovinocultura Mato-grossense.

Assim como observado neste estudo, em outros levantamentos realizados no Brasil, independente da aptidão das raças que predominam na região estudada (corte ou leite) a maioria das mortalidades de bovinos está associada às doenças que cursam com sinais neurológicos ou ao consumo de plantas tóxicas (Casagrande et al. 2008, Galiza et al. 2010, Lucena et al. 2010, Ribas et al. 2013). Dessa forma, pode-se sugerir que as principais causas de mortalidades em sistemas extensivos de criação de bovinos no Brasil estão relacionadas a problemas nas pastagens como invasão por plantas, baixa digestibilidade, suplementação mineral inadequada, ou ainda à falha no manejo sanitário como controle de vetores ou vacinação inadequada.

Neste estudo as doenças que cursam com sinais clínicos neurológicos (DSCN) foram responsáveis pela maior parte da mortalidade de bovinos no estado, totalizando 252 casos $(22,4 \%)$, como pôde ser observado nos Quadros 3-5. Dentre esses casos foram frequentes as doenças causadas por agentes infecciosos como raiva (88 casos), BoHV-5 (42 casos) e encefalites linfoplasmocíticas de causa não determinada (30 casos). Das DSCN que não são de origem infecciosa destaca-se a polioencefalomalacia (32 casos) e botulismo (31 casos). Estudos conduzidos na Paraíba (Assis et al. 2010, Galiza et al. 2010) e em Mato Grosso do Sul (Ribas et al. 2013) também relatam as DSCN como as causas mais importantes de mortes de bovinos, enquanto na região sul do Brasil estudos realizados em Santa Catarina (Casagrande et al. 2008) e no Rio Grande do Sul (Lucena et al. 2010) apontam as intoxicações por plantas como maiores responsáveis por mortes de bovinos. As DSCN assumiram grande importância após o diagnóstico da encefalopatia es-

Quadro 2. Classificações das doenças diagnosticadas em bovinos no LPV-UFMT entre 2005 a 2014 e distribuição por idade em meses dos animais acometidos

\begin{tabular}{|c|c|c|c|c|c|c|c|c|c|c|c|}
\hline Classificação & $0-6$ & $6-12$ & $12-18$ & $18-24$ & 24-30 & $30-36$ & $36-42$ & $48-54$ & $>54$ & NI & Total \\
\hline Inconclusivo & 58 & 39 & 66 & 47 & 56 & 5 & 25 & 10 & 60 & 205 & 571 \\
\hline Doenças inflamatórias e parasitárias & 75 & 27 & 31 & 28 & 42 & 1 & 26 & 13 & 24 & 42 & 309 \\
\hline Doenças tóxicas e toxiinfecções & 3 & 9 & 8 & 9 & 19 & 0 & 11 & 3 & 25 & 23 & 110 \\
\hline Neoplasmas e lesões tumoriformes & 0 & 0 & 1 & 0 & 0 & 1 & 5 & 1 & 23 & 13 & 44 \\
\hline Doenças degenerativas & 1 & 0 & 9 & 3 & 5 & 1 & 4 & 2 & 4 & 5 & 34 \\
\hline Distúrbios por agentes físicos & 3 & 4 & 1 & 1 & 3 & 0 & 1 & 1 & 12 & 6 & 32 \\
\hline Distúrbios metabólicos e nutricionais & 1 & 4 & 1 & 0 & 3 & 0 & 0 & 1 & 1 & 5 & 16 \\
\hline Outras categorias & 0 & 0 & 0 & 1 & 2 & 4 & 1 & 0 & 0 & 0 & 8 \\
\hline Total por idade em meses & 133 & 84 & 117 & 89 & 131 & 12 & 76 & 31 & 151 & 303 & 1124 \\
\hline
\end{tabular}


pongiforme bovina (BSE) na Inglaterra (Wells et al. 1987) e da possível relação dessa doença com a nova variante da doença de Creutzfeldt-Jakob (DCJ) em humanos (Will et al. 1996, Almond \& Pattison 1997). 0 aumento da eficiência da vigilância sanitária sobre as DSCN, devido à preocupação com o diagnóstico dessas duas novas doenças, estimulou o registro de doenças já existentes e o relato de novas que

Quadro 3. Doenças inflamatórias e parasitárias de bovinos diagnosticadas no período de 2005 a 2014 no LPV-UFMT

\begin{tabular}{|c|c|c|}
\hline Doenças inflamatórias e parasitárias & Freq. Absoluta & Freq. Relativa \% \\
\hline Raiva & 88 & 28,47 \\
\hline BoHV-5* & 42 & 13,59 \\
\hline $\begin{array}{l}\text { Encefalite linfoplasmocítica de } \\
\text { etiologia não definida }\end{array}$ & 30 & 9,70 \\
\hline Pneumonia bacteriana & 14 & 4,53 \\
\hline Enterite por Hobbi-Like vírus & 10 & 3,23 \\
\hline Meningoencefalite purulenta & 10 & 3,23 \\
\hline Tristeza parasitária & 9 & 2,91 \\
\hline Febre catarral maligna & 8 & 2,58 \\
\hline Estomatite vesicular & 8 & 2,58 \\
\hline Carbúnculo sintomático & 7 & 2,26 \\
\hline Hemoncose & 6 & 1,94 \\
\hline Septicemia & 6 & 1,94 \\
\hline Mastite & 4 & 1,29 \\
\hline Eimeriose & 4 & 1,29 \\
\hline Granuloma parasitário & 3 & 0,97 \\
\hline Granuloma vacinal & 3 & 0,97 \\
\hline Broncopneumonia bacteriana & 3 & 0,97 \\
\hline Dermatofilose & 3 & 0,97 \\
\hline Abcessos & 3 & 0,97 \\
\hline Enterite bacteriana & 3 & 0,97 \\
\hline Dermatite digital & 2 & 0,64 \\
\hline Endocardite valvular & 2 & 0,64 \\
\hline Enterite por rotavírus & 2 & 0,64 \\
\hline Onfaloflebite & 2 & 0,64 \\
\hline Salmonelose & 2 & 0,64 \\
\hline Pneumonia Intersticial & 2 & 0,64 \\
\hline Poliartrite purulenta & 2 & 0,64 \\
\hline Peritonite & 2 & 0,64 \\
\hline Cisticercose & 2 & 0,64 \\
\hline Pitiose & 2 & 0,64 \\
\hline Pneumonia necrótica & 2 & 0,64 \\
\hline Nefrite tromboembólica & 2 & 0,64 \\
\hline Dermatite micótica & 1 & 0,32 \\
\hline Estomatite bacteriana & 1 & 0,32 \\
\hline Colibacilose neonatal & 1 & 0,32 \\
\hline Bursite granulomatosa & 1 & 0,32 \\
\hline Embolia bacteriana e cisticercose & 1 & 0,32 \\
\hline Hepatite necrótica & 1 & 0,32 \\
\hline Hepatite eosinofílica & 1 & 0,32 \\
\hline Linfadenite granulomatosa & 1 & 0,32 \\
\hline Meningite & 1 & 0,32 \\
\hline Encefalopatia hepática & 1 & 0,32 \\
\hline BRSV** & 1 & 0,32 \\
\hline Micotoxicose aguda & 1 & 0,32 \\
\hline Miocardite de etiologia não determinada & 1 & 0,32 \\
\hline Outras verminoses & 1 & 0,32 \\
\hline Placentite bacteriana & 1 & 0,32 \\
\hline Pleurite fibrinosa & 1 & 0,32 \\
\hline Pneumonia micótica & 1 & 0,32 \\
\hline Pneumonia verminótica & 1 & 0,32 \\
\hline Polisserosite por E. coli & 1 & 0,32 \\
\hline Toxemia puerperal & 1 & 0,32 \\
\hline Tuberculose & 1 & 0,32 \\
\hline Total & 309 & 100,00 \\
\hline
\end{tabular}

* BoHV-5 = Herpes vírus bovino tipo $5,{ }^{* *} \mathrm{BRSV}=$ Vírus sincicial respiratório bovino.
Quadro 4. Neoplasmas, lesões tumoriformes, doenças degenerativas e doenças tóxicas e toxiinfecções em bovinos diagnosticadas no LPV-UFMT no período de 2005 a 2014

\begin{tabular}{|c|c|c|}
\hline Neoplasmas e lesões tumoriformes & Freq. Absoluta & Freq. Relativa \% \\
\hline Carcinoma de células escamosas & 19 & 43,18 \\
\hline Linfoma & 5 & 11,10 \\
\hline Papiloma & 3 & 6,82 \\
\hline Fibroma interdigital & 2 & 4,55 \\
\hline Melanoma & 2 & 4,55 \\
\hline Mesotelioma & 2 & 4,55 \\
\hline Schwanoma & 2 & 4,55 \\
\hline Tecido de granulação & 2 & 4,55 \\
\hline Hemangioma de prepúcio & 1 & 2,27 \\
\hline Carcinoma de útero & 1 & 2,27 \\
\hline Chifre cutâneo & 1 & 2,27 \\
\hline Meningioma & 1 & 2,27 \\
\hline Cisto folicular dermóide & 1 & 2,27 \\
\hline Mixoma fibroso & 1 & 2,27 \\
\hline Feocromocitoma & 1 & 2,27 \\
\hline Total & 44 & 100,0 \\
\hline Doenças Degenerativas & Freq. Absoluta & Freq. Relativa \% \\
\hline Polioencefalomalacia & 32 & 94,12 \\
\hline Glomerulonefrite esclerosante & 1 & 2,94 \\
\hline Osteocondrose & 1 & 2,94 \\
\hline Total & 34 & 100,00 \\
\hline Doenças Tóxicas e toxiinfecções & Freq. Absoluta & Freq. Relativa \% \\
\hline Botulismo & 31 & 28,18 \\
\hline Intoxicação por Brachiaria spp. & 20 & 18,18 \\
\hline Necrose hepatocelular & 17 & 15,45 \\
\hline Anemia hemolítica tóxica & 10 & 9,09 \\
\hline Fibrose hepática & 5 & 4,54 \\
\hline Intoxicação por Palicourea marcgravii & 4 & 3,63 \\
\hline Intoxicação por Senna ocidentallis & 5 & 4,54 \\
\hline Calcinose enzoótica & 4 & 3,63 \\
\hline Necrose renal tubular tóxica & 3 & 2,72 \\
\hline Intoxicação por ionóforo & 2 & 1,81 \\
\hline Intoxicação por abamectina & 2 & 1,81 \\
\hline Tétano & 2 & 1,81 \\
\hline $\begin{array}{l}\text { Intoxicação cianogênica por Capim } \\
\text { do gênero Cynodon }\end{array}$ & 1 & 0,90 \\
\hline $\begin{array}{l}\text { Intoxicação por Stryphnodendron } \\
\text { fissuratum }\end{array}$ & 1 & 0,90 \\
\hline Intoxicação por Chumbo & 1 & 0,90 \\
\hline Intoxicação por Ipomoea fistulosa & 1 & 0,90 \\
\hline Intoxicação por Amorimia pubiflora & 1 & 0,90 \\
\hline Total & 110 & 100,00 \\
\hline
\end{tabular}

antes não eram diagnosticadas (Barros \& Marques 2003), bem como o cumprimento das exigências sanitárias internacionais (Barros \& Marques 2003).

A raiva foi a doença mais frequentemente diagnosticada. Referente a esta doença, ressalta-se a importância da parceria com o órgão de defesa agropecuária do Estado (INDEA) tanto na confirmação do diagnóstico como na sua exclusão, através da realização da técnica de imunofluorescência direta. A Raiva tem caráter cíclico (Lucena et al. 2010), tendo em vista que o seu desaparecimento não representa controle efetivo da doença (Mori 1998). Vários outros estudos apontam a raiva como uma das principais doenças de bovinos no Brasil (Sanches et al. 2000, Lucena et al. 2010, Galiza et al. 2010, Ribas et al. 2013). Esta pode ser considerada uma doença em reemergência, possivelmente em razão do relaxamento nas medidas de controle do morcego Desmodus rotundus (MAPA, 2009) e da negligência no calendário vacinal.

Infecção por BoHV-5 (42 casos) é uma das principais 
Quadro 5. Distúrbios causados por agentes físicos, distúrbios metabólicos e nutricionais e outras categorias de doenças de bovinos diagnosticados no LPV-UFMT em 2005 a 2014

\begin{tabular}{|c|c|c|}
\hline Distúrbios causados por agentes físicos & Freq. Absoluta & Freq. Relativa $\%$ \\
\hline $\begin{array}{l}\text { Obstrução intestinal por } \\
\text { fitobenzoares (Stylosanthes sp) }\end{array}$ & 7 & 21,88 \\
\hline Compressão medular & 5 & 15,63 \\
\hline Fratura & 5 & 15,63 \\
\hline Compactação ruminal & 3 & 9,38 \\
\hline Parto distócico & 3 & 9,38 \\
\hline $\begin{array}{l}\text { Obstrução intestinal por } \\
\text { piloconcremento }\end{array}$ & 2 & 6,25 \\
\hline Miopatia por compressão & 2 & 6,25 \\
\hline Intussuscepção intestinal & 1 & 3,13 \\
\hline Obstrução esofágica por corpo estranho & 1 & 3,13 \\
\hline Traumatismo craniano & 1 & 3,13 \\
\hline Pneumonia aspirativa & 1 & 3,13 \\
\hline Retículo peritonite traumática & 1 & 3,13 \\
\hline Total & 32 & 100,00 \\
\hline Distúrbios metabólicos e nutricionais & Freq. Absoluta & Freq. Relativa \% \\
\hline Urolitíase & 5 & 31,25 \\
\hline Timpanismo & 4 & 25,00 \\
\hline Osteomalacia/ raquitismo & 2 & 12,50 \\
\hline Deficiência de cobalto & 1 & 6,25 \\
\hline Deficiência de cobre & 1 & 6,25 \\
\hline Hipocalcemia & 1 & 6,25 \\
\hline Lipidose hepática & 1 & 6,25 \\
\hline Acidose ruminal & 1 & 6,25 \\
\hline TOTAL & 16 & 100,00 \\
\hline Outras categorias & Freq. Absoluta & Freq. Relativa \% \\
\hline Inversão térmica & 4 & 50,00 \\
\hline Hipoplasia pulmonar & 1 & 12,05 \\
\hline Necrose de cauda & 1 & 12,05 \\
\hline Úlcera de abomaso & 1 & 12,05 \\
\hline Teleangiectasia & 1 & 12,05 \\
\hline Total & 8 & 100,00 \\
\hline
\end{tabular}

causas de encefalopatia em bovinos no Brasil, sobretudo na região Centro-Oeste (Colodel et al. 2002, Arruda et al. 2010, Ribas et al. 2013). A doença afeta mais frequentemente bovinos jovens e tem sido detectada em várias regiões (Riet-Correa et al. 1989, Sanches et al. 2000, Claus et al. 2007, Galiza et al. 2010, Lucena et al. 2010). Maiores detalhes sobre esta doença em Mato Grosso são fornecidos em estudos anteriores (Colodel et al. 2002, Arruda et al. 2010).

Neste estudo verificou-se que, aproximadamente, $1 \mathrm{em}$ cada 8 casos de bovinos com DSCN, o diagnóstico etiológico não pôde ser definido, permanecendo apenas o diagnóstico morfológico de meningoencefalite linfoplasmocítica de etiologia não definida (30 casos). Levando-se em conta apenas as DSCN que são compatíveis com este diagnóstico morfológico, pode-se afirmar que 1 em cada 6 bovinos permanece sem diagnóstico etiológico definido. Meningoencefalites linfoplasmocíticas de etiologia não definida foram frequentemente observadas em outros estudos envolvendo diferentes regiões do Brasil (Sanches et al. 2000, Galiza et al. 2010, Lucena et al. 2010, Ribas et al. 2013). As causas mais prováveis para essa lesão em bovinos no Brasil são doenças virais como febre catarral maligna, Raiva e BoHV5. Embora febre catarral maligna possa causar meningoencefalite linfoplasmocítica no SNC de bovinos, a vasculite é o aspecto distintivo das lesões, pois acontecem em outros órgãos e não só no SNC (Rech et al. 2005, Mendonça et al. 2008, Furlan et al. 2012). Desse modo, raiva e encefalite por BoHV-5 constituiriam os dois principais diferenciais para o diagnóstico nesses casos.

Dezesseis dos 30 casos com meningoencefalite linfoplasmocítica de etiologia não definida foram encaminhados para exame histopatológico pelo Laboratórório de Apoio a Saúde Animal do Instituto de Defesa Agropecuária de Mato Grosso (LASA/INDEA, MT), uma vez que foram negativos para raiva através de imunofluorescência direta (IFD) e inoculação intracerebral em camundongos. Nesses casos não foram observados corpúsculos de Negri, característicos da raiva, bem como não houve marcação positiva para antígeno viral por imunoistoquímica. Embora a correlação entre esses testes seja alta (Pedroso et al. 2009, Achkar et al. 2010, Marcolongo-Pereira et al. 2011, Ribas et al. 2013), falsos negativos podem ocorrer ( Silva et al. 1974) principalmente quando leva-se em conta as concentrações virais em diferentes fragmentos do SNC (Carrieri et al. 2006). Os outros 14 casos foram encaminhados para diagnósticos por veterinários de campo e consistiam de amostras fixadas em formol o que impossibilita a realização da técnica de IFD para raiva, aumentando assim a chance de falsos negativos. No tocante ao BoHV-5 constituem lesões histológicas que caracterizam a doença, além da meningoencefalite linfoplasmocítica, a necrose neuronal acompanhada de edema do neurópilo perineuronal e da substância branca com ocasionais inclusões intranucleares eosinofílicas em astrócitos e neurônios (Riet-Correa et al. 1989, Colodel et al. 2002, Elias et al. 2004). No entanto, a localização e intensidade dessas lesões são mais acentuadas na região rostral do telencéfalo, de modo que, caso essa região não seja coletada, as lesões podem ser inespecíficas e caracterizadas por meningoencefalite linfoplasmocítica. Esse fato é demonstrado por Arruda et al. (2010), em um estudo molecular envolvendo casos com diagnóstico morfológico de meningoencefalite linfoplasmocítica de etiologia não definida onde observou-se 33\% de positividade para BoHV-5.

Neste estudo a ocorrência de 10 casos $(3,23 \%)$ de enterite por Hobi-like vírus em bezerros na mesorregião Centro-Sul de Mato Grosso no período de 2011 a 2013 (Campos et al. 2014), indica que a enfermidade é emergente no estado e deve entrar no diagnóstico diferencial de doenças entéricas em bovinos (Cortez et al. 2006, Bianchi et al. 2011, Rodrigues et al. 2011, Bauermann et al. 2013, Weber et al. 2014, Bauermann et al. 2015, Silveira et al 2015) . Dos 309 casos pertencentes ao grupo das doenças inflamatórias e parasitárias, $23(7,42 \%)$ tinham parasitose como principal condição. Dentre as doenças parasitárias, as mais relevantes foram tristeza parasitária bovina ( 9 casos) e haemoncose ( 6 casos). Dos casos de TPB, 5 bovinos tinham idade até 8 meses, sugerindo que a queda da imunidade passiva partir do 28을 dia após o nascimento poderia relacionar-se ao aumento da ocorrência dessa doença (Madruga et al. 1984), uma vez que a enfermidade é verificada com maior frequência em bezerros com idade superior a 4 meses (Madruga et al. 1986).

O segundo grupo de doenças mais observadas no estado estão agrupadas na categoria de doenças tóxicas e toxiinfecções. Dentre elas podemos ressaltar o botulismo (31 casos), intoxicação por Brachiaria spp. (20 casos), necro- 
se e degeneração hepatocelular aguda (17 casos), anemia hemolítica tóxica (10 casos) e intoxicação por Palicourea marcgravii (5 casos).

Os diagnósticos de botulismo foram realizados com base no quadro clínico e epidemiologia e na ausência de lesões macroscópicas e histopatológicas (Dutra et al. 2005, Galiza et al. 2010). Clinicamente os animais apresentaram decúbito esternal permanente, paralisia flácida dos membros e cauda, relaxamento da mandíbula e flacidez de língua. A principal forma de botulismo foi a epizoótica associada à osteofagia frequentemente relacionada com carência de fósforo na pastagem e suplementação inadequada de mineral sendo esta doença considerada uma importante causa de morte na pecuária extensiva (Dutra et al. 2005). Bovinos com idade de 1 a 9 anos foram afetados indicando que todas as categorias podem apresentar a doença.

A intoxicação por Brachiaria spp. foi observada em forma de surtos tanto no período de seca como de chuva. Em 9 dos 20 surtos o principal sinal clínico foi a fotossensibilização hepatógena, sendo uma importante causa de prejuízos econômicos em bovinos no Mato Grosso. Bovinos de diversas faixas etárias foram afetados. A maioria dos surtos foi verificada em bovinos de até 24 meses colaborando com outros estudos que mencionam que a maior parte dos surtos de fotossensibilização ocorrem em bovinos de até 2 anos de idade, sendo, portanto, os mais susceptíveis (Tokarnia et al. 2012).

Necrose e degeneração hepatocelular aguda tiveram como suspeita principal o consumo de plantas hepatotóxicas, a causa mais comum de necrose hepatocelular foi relacionada ao consumo de folhas de Pterodon emarginatus (Cruz et al. 2012). Há ocorrência de Vernonia rubricaulis em áreas limítrofes do Pantanal no Estado de Mato Grosso, esta planta é potencialmente hepatotóxica (Brum et al. 2002) mas apesar de evidencias da ocorrência, não há registros dessa intoxicação no LPV-UFMT.

Anemias hemolíticas tóxicas tiveram um aumento expressivo do número de casos nos 5 últimos anos, sendo que os casos citados neste estudo se referiram a surtos em propriedades onde as mortes acometeram sempre mais de um bovino. Os sinais clínicos observados incluem fraqueza, incoordenação motora, icterícia, urina enegrecida e morte de bovinos principalmente quando movimentados. Entre os achados microscópicos, no fígado, nota-se necrose centrolobular e paracentral acentuada, nos rins degeneração e necrose tubular multifocal acentuada, as células tubulares estavam com citoplasma vacuolizado e tumefeito com gotículas hialinas e acúmulo de material fortemente hialino (nefrose hemoglobinúrica) na luz tubular. As evidências clínicas, morfológicas e laboratoriais permitem afirmar que doenças como leptospirose, anaplasmose, babesiose, tripanossomíase, hemoglobinúria bacilar e hematúria enzoótica não foram a causas dessa mortalidade. Investigando-se as áreas de ocorrência da doença, não foram encontradas plantas conhecidas como causa de hemoglobinúria tóxica como Brachiaria radicans, Ditaxis desertorum, Indigofera suffruticosa e Allium sp., relatadas por Tokarnia et al. (2012). Para elucidação da causa serão necessários estudos mais aprofundados.
Embora seja a planta tóxica mais importante do grupo das que causam "morte súbita" e ainda a planta tóxica mais importante do Brasil (Tokarnia et al. 2012), a intoxicação por Palicourea marcgravii teve uma frequência relativa em torno de $4 \%$ dos casos de intoxicações descritos nesse estudo. Contudo acredita-se em subnotificação, similar ao que ocorre em casos de intoxicação por Amorimia pubiflo$r a$ (Becker et al. 2013). Possivelmente isso ocorre devido ao conhecimento sobre a planta e seus efeitos, pelos veterinários, proprietários e vaqueiros, que ocasionalmente informam ocorrências, mas não se faz necropsia ou outros exames para complementação diagnóstica. As notificações registradas no LPV-UFMT ocorreram em pastagens recém-abertas que anteriormente eram áreas de florestas e por proprietários oriundos de outras regiões que recentemente adquiriram fazendas e, portanto, desconheciam P. marcgravii.

Neoplasmas e lesões tumoriformes foram identificadas em 4\% (44/1124) de todos os bovinos examinados post-mortem. Estes valores estão abaixo dos encontrados em outro estudo brasileiro $(8,7 \%)$ realizado no Sul do Brasil (Lucena et al. 2010) e no Canadá (7\%) (Waldner et al. 2009). A baixa ocorrência em relação aos outros estados brasileiros pode estar relacionada à aptidão, com predomínio de bovinocultura de corte no estado de Mato Grosso, com 90,57\% (740/817) das raças encontradas neste estudo, sendo estas a Nelore $(74,48 \%)$, mestiça $(20,40 \%)$, Caracu $(0,94 \%)$, Brahman $(0,67 \%)$, Brangus $(0,67 \%)$, Blonde D'aquitaine $(0,40 \%)$, Senepol $(0,27 \%)$ e Canchim $(0,13 \%)$, todas de corte, do qual a idade de abate ou taxa de renovação do rebanho é de 2,0 a 2,5 anos. Dessa forma há menor ocorrência de neoplasmas quando comparado a bovinos de leite, que foram $9,42 \%$ das raças encontradas neste estudo, sendo estas Girolando (44,15\%), Holandesa $(23,37 \%)$, Gir (22,07\%), Simental (5,19\%), Pardo Suíço (2,59\%), Jersey $(1,29 \%)$ e Rubia Gallega $(1,29 \%)$, uma vez que estes últimos permanecem na propriedade até uma idade mais avançada. 0 tipo mais comum observado foi o carcinoma de células escamosas, o qual acredita-se ter relação com exposição crônica à radiação ultravioleta (UV) (Burdin 1964, Wettimuny 1974, MacLachlan \& Kennedy 2002, Ginn et al. 2007). Esta neoplasia foi mais frequente na região ocular $(37,8 \%)$, como observado por Ribeiro et al. (2014) em bovinos abatidos no norte do estado de Mato Grosso, e na base de chifre $(4,4 \%)$, observado exclusivamente em bovinos da raça nelore com idade superior a 5 anos corroborando com os achados de Lemos \& Nakazato (2007).

0 segundo tipo de neoplasma mais reportado foi o linfoma $(11,10 \%)$ sendo observado em vacas de 5 a 8 anos de idade. Embora o vírus da leucose esteja amplamente distribuído em rebanhos bovinos, a frequência de neoplasias foi baixa. Este dado corrobora com outros estudos realizados uma vez que cerca de $30 \%$ dos animais infectados pelo vírus da leucemia bovina desenvolvem linfocitose persistente (LP) e apenas 0,1 a $0,5 \%$ dos animais infectados, com idade entre 4 e 8 anos, desenvolvem a forma tumoral, caracterizando a forma clínica da doença (Ferrer 1980, Burny et al. 1985, Johnson \& Kaneene 1992, Braga \& Van der Laan 2001). 
Dentre as doenças degenerativas, a polioencefalomalacia (PEM) foi a mais prevalente e $94,12 \%(32 / 34)$ dos casos ocorreram em bovinos de até 3 anos de idade. Não se notou sazonalidade na ocorrência dessa doença, a qual foi observada em $41,17 \%(14 / 34)$ dos casos em época da seca, e em $47,05 \%$ (16/34) dos casos na época da chuva quando há boa disponibilidade de pastagem e suplementação, como relatado por Galiza et al. (2010). Dos 32 casos observados, 31 ocorreram em propriedades com sistema extensivo de criação e um caso foi em sistema de confinamento. Assim como observado neste estudo, a doença ocorre mais frequentemente em bovinos criados em regime extensivos (Riet-Correa et al. 1989, Riet-Correa \& Schild 1995, Colodel et al. 2002, Gomes et al. 2002, Elias et al. 2004). A etiologia da PEM não é totalmente estabelecida, no entanto, ela é frequentemente associada à deficiência de tiamina, intoxicação por enxofre, intoxicação por sal (ou privação de água) e intoxicação por chumbo (Sant'Ana et al. 2009a, Sant'Ana et al. 2009b). Neste estudo a etiologia da PEM não pôde ser determinada, entretanto, não foram anotadas evidências de intoxicação por chumbo, bem como não se encontrou, nos históricos, condições condizentes com privação de água ou intoxicação por sal.

Distúrbios causados por agentes físicos foram observados em 2,84\% (32/1124) dos casos, dentre eles ressaltam-se a obstrução intestinal por fitobenzoares associado ao consumo de pastagens de Stylosanthes sp. diagnosticado em $21,88 \%(7 / 32)$ e a compressão medular associada a aplicação da vacina de febre aftosa em 15,63\% (5/32). Esses casos foram descritos anteriormente (Ubiali et al. 2011, Ubiali et al. 2013).

Doenças nutricionais e metabólicas foram pouco observadas neste estudo, concordado com dados descritos no Sul do Brasil (Lucena et al. 2010). Entretanto, chama a atenção que todos os casos de timpanismo contabilizados neste estudo ocorreram na mesma propriedade com sistema de confinamento e foram relacionados com a administração de dieta hipercalórica que levou ao desenvolvimento dos sinais clínicos. Este dado foi similar ao observado em outros países com sistema de criação intensiva (Watson et al. 2008, Waldner et al. 2009).

Dos distúrbios classificados em outras categorias, a hipotermia de bovinos associada à inversão térmica foi a doença mais comum registrada no LPV-UFMT, e tem características similares às descritas por Santos et al. (2012). Os casos foram caracterizados por mortalidade de bovinos zebuínos, com escore de condição corporal baixa e ausência de alterações macro ou microscópicas significativas. São importantes para o diagnóstico desta entidade os aspectos epidemiológicos como: morte de bovinos azebuados imediatamente após quedas bruscas de temperatura, aumento da intensidade de ventos, precipitação pluviométrica e falta de abrigos naturais ou artificiais para proteção dos bovinos contra essas intempéries.

No presente trabalho, o índice de diagnósticos não conclusivos $(50,71 \%$ ou $570 / 1124)$ foi considerado alto quando comparada com outros trabalhos realizados no Brasil (Casagrande et al. 2008, Lucena et al. 2010). Como um dos fatores para esta discrepância relacionou-se a distância en- tre as propriedades e o laboratório associada à coleta inadequada das amostras. Dentre os casos com diagnósticos não conclusivos, $82,98 \%(473 / 570)$ corresponderam às amostras encaminhadas por veterinários de campo. Muitas dessas amostras chegam ao laboratório, congeladas ou em temperatura ambiente sem fixação adequada, ou ainda em frascos pequenos contendo pouco formol e com fragmentos de órgãos espessos. A restrição ao comercio de formol e a longa distância, portanto interferem na qualidade das amostras antes da análise no laboratório. Outro fator importante a ser ressaltado é a importância da associação entre fatores epidemiológicos e clínicos com a adequada interpretação dos achados de necropsia para complementação diagnóstica. Notou-se no decorrer destes 10 anos de análise de amostras, constantes inconsistências. Acredita-se que muitos destes diagnósticos inconclusivos estão relacionados ao envio insuficiente de amostras e ou coleta inadequada de dados clínicos e epidemiológicos, uma vez que, com muita frequência, para achados anatômicos, lesões incidentais e alterações post mortem foi dado importância diagnóstica negligenciando-se a coleta adequada e diminuindo, portanto, a acurácia diagnóstica.

Este trabalho relaciona as principais doenças que acometem bovinos no Estado de Mato Grosso, com base nas alterações morfológicas, permitindo verificar que algumas delas, como a raiva, está também presente de forma expressiva em outros estados brasileiros. As informações acima geradas poderão servir de base para a pratica de ensino em medicina veterinária, além de orientar médicos veterinários de campo sobre a frequência das principais doenças diagnosticadas em bovinos no Estado, bem como ajudar na realização de diagnósticos diferenciais.

\section{REFERÊNCIAS}

Achkar S.M., Fernandes E.R., Carrieri M.L., Castro A.B.M., Batista A.M., Duarte M.I.S. \& Kotait. I. 2010. Sensibilidade da técnica de imunohistoquímica em fragmentos de sistema nervoso central de bovinos e equinos naturalmente infectados pelo vírus da raiva. Pesq. Vet. Bras. 30(3):211-218.

ACRIMAT 2012. Diretrizes para o desenvolvimento da pecuária de corte de Mato Grosso, Visão de Universitários e Pecuaristas. Associação dos Criadores de Mato Grosso. 23p. Disponível em <http://www.acrimat. org.br/novo/arquivos/guias_cartilhas/22012014032026.pdf> Acesso em 9 jun. 2013.

ACRIMAT 2015. Informativo Mensal da Associação dos Criadores de Mato Grosso. Ed. 52, Ano 7, jan. Disponível em <http://www.acrimat.org.br/ novo/arquivos/informativos/09022015120105.pdf> Acesso em 14 mai. 2015.

Almond J. \& Pattison J. 1997. Human BSE. Nature 389:437-38.

Arruda L.P., Nakazato L., Dutra V., Lemos R.A.A., Nogueira A.P.A., Cruz R.A.S., Pescador C.A. \& Colodel E.M. 2010. Detecção molecular de herpesvírus bovino 1 e 5 em amostras de encéfalo conservadas em formol e emblocadas em parafina provenientes de bovinos com doença neurológica. Pesq. Vet. Bras. 30(8):646-650.

Assis T.S., Medeiros R.M.T., Riet-Correa F., Galiza G.J.N., Dantas A.F.M. \& Oliveira D.M. 2010. Intoxicações por plantas diagnosticadas em ruminantes e equinos e estimativa das perdas econômicas na Paraíba. Pesq. Vet. Bras. 30(1):13-20.

Bauermann F.V., Falkenberg S.M., Decaro N., Flores E.F. \& Ridpath J.F. 2015. Experimental infection of calves, sheep, goats and pigs with HoBi-like viruses by direct inoculation or exposure to persistently infected calves. Vet. Microbiol. 181(3):289-293. 
Bauermann F.V., Ridpath J.F., Weiblen R. \& Flores E.F. 2013. HoBi-like viruses an emerging group of pestiviruses. J. Vet. Diagn. Invest. 25(1):6-15.

Barros C.S.L. \& Marques G.H.F. 2003. Procedimentos para o diagnóstico das doenças do sistema nervoso central de bovinos. MAPA/SDA/DDA, Brasília. 50p.

Becker M., Caldeira F.H.B., Carneiro F.M., Oliveira L.P., Tokarnia C.H., Riet-Correa F., Lee S.T. \& Colodel E.M. 2013. Importância da intoxicação por Amorimia pubiflora (Malpighiaceae) em bovinos em Mato Grosso: reprodução experimental da intoxicação em ovinos e bovinos. Pesq. Vet. Bras. 33(9):1049-1056.

Bianchi E., Martins M., Weiblen R. \& Flores E.F. 2011. Perfil genotípico e antigênico de amostras do vírus da diarréia viral bovina isoladas no Rio Grande do Sul (2000-2010). Pesq. Vet. Bras. 31(8):649-655.

Braga F.M. \& Van der Laan C.W. 2001. Leucose enzoótica bovina, p.126134. In: Riet-Correa F., Schild A.L., Méndez M.D.C. \& Lemos R.A.A. (Eds), Doenças de Ruminantes e Equinos. Varela, São Paulo.

Brum K.B., Purisco E., Lemos R.A.A. \& Riet-Correa F. 2002. Intoxicação por Vernonia rubricaulis em bovinos no Mato Grosso do Sul. Pesq. Vet. Bras. 22(3):119-128.

Burdin M.L. 1964. Squamous carcinoma of the vulva of cattle in Kenya. Res. Vet. Sci. 5:497-505.

Burny A., Bruck C., Cleute Y., Couez D., Deschamps J., Gregoire D., Ghysdael J., Kettmann R., Mammerickx M., Marbaix G. \& Portelle D. 1985. Bovine leukaemia virus and enzootic bovine leukosis. Onderstepoort J. Vet. Res. 52:133-144.

Campos C.G., Lopes L.L., Azevedo J.B., Silva L.A., Bezerra K.S., Silveira S., Canal C.W. \& Pescador C.A. 2014. Enterite viral por Pestivírus HoBi-like em bezerros no município de Cáceres, Mato Grosso, Brasil. Anais II Encontro Internacional de Sanidade de Animais de Produção (Enisap) e VII Encontro Nacional de Diagnóstico Veterinário (Endivet), Cuiabá, MT.

Carrieri M.L., Peixoto Z.M.P., Paciencia M.L.B., Kotait I. \& Germano P.M.L. 2006. Laboratory diagnosis of equine rabies and its implications for human postexposure prophylaxis. J. Virol. Methods 2:1-9.

Casagrande R.A., Mazzoco M.B., Frizon R., Lentz D., Traverso S.D. \& Gava A. 2008. Doenças de bovinos diagnosticadas pelo Laboratório de Patologia Animal CAV/UDESC de Janeiro de 2000 a Abril de 2008. Anais VI Encontro Nacional de Diagnóstico Veterinário (Endivet), Campo Grande, MS.

Claus M.P., Alfieri A.F., Médici K.C., Lunardi M. \& Alfieri A.A. 2007. Bovine herpes-vírus 5 detection by vírus isolation in cell culture and Multiplex-PCR in central nervous system from cattle with neurological disease in Brazilian herds. Braz. J. Microbiol. 38:485-490.

Colodel E.M., Nakazato L., Weiblen R., Mello R.M., Silva R.P., Souza M.A., Oliveira J.A. \& Caron L. 2002. Meningoencefalite necrosante em bovinos causada por herpesvírus bovino no estado de Mato Grosso, Brasil. Ciência Rural 32(2):293-298.

Conab 2014. Indicadores da Agropecuária: quadro de suprimentos. Companhia Nacional de Abastecimento. Disponível em <http://www.conab. gov.br/conteudos.php?a=1470\&t=2> Acesso em 10 jan. 2015.

Cortez A., Heinemann M.B., Castro A.M.M., Soares R.M., Pinto A.M.V., Alfieri A.A., Flores E.F., Leite R.C. \& Richtzenhain L.J. 2006. Genetic characterization of Brazilian bovine viral diarrhea virus isolates by partial nucleotide sequencing of the 5'-UTR region. Pesq. Vet. Bras. 26(4):211-216.

Cruz R.A.S., Oliveira L.P., Caldeira F.H.B., Mendonça F.S., Bacha F.B., Pott A., Lemos R.A.A. \& Colodel E.M. 2012. Intoxicação espontânea e experimental por Pterodon emarginatus (Fabaceae Faboideae) em bovinos e experimental em ovinos. Pesq. Vet. Bras. 32(11):1087-1094.

Dutra I.S., Döbereiner J. \& Sousa A.M. 2005. Botulismo em bovinos de corte e leite alimentados com cama de frango. Pesq. Vet. Bras. 25(2):115-119.

Elias F., Schild A.L. \& Riet-Correa F. 2004. Meningoencefalite e encefalomalacia por Herpes vírus bovino-5 (BHV-5): distribuição das lesões no sistema nervoso central de bovinos naturalmente infectados. Pesq. Vet. Bras. 24(3):123-131.

Ferrer J.F. 1980. Bovine lymphosarcoma. Adv. Vet. Sci. Comp. Med. 24:2-65.

Furlan F.H., Amorim T.M., Justo R.V., Mendes E.R.S., Zilio M.G., Costa F.L., Nakazato L. \& Colodel E.M. 2012. Febre catarral maligna em bovinos no norte de Mato Grosso, Brasil. Acta Scient. Vet. 40(2):1043.
Gaeti J.G.L.N., Lana M.V.C., Silva G.S., Lopes L.L., Campos C.G., Harumi F., Colodel E.M., Costa E.F., Corbellini L.G., Nakazato L. \& Pescador C.A. 2014. Ureaplasma diversum as a cause of pustular vulvovaginitis in bovine females in Vale Guaporé, Mato Grosso state, Brazil. Tróp. Anim. Health Prod. 46(6):1065.

Gagea M.I., Bateman K.G., Dreumel T.V., McEwen B.J., Carman S., Archambault M., Shanahan R.A. \& Caswell J.L. 2006. Diseases and pathogens associated with mortality in Ontario beef feedlots. J. Vet. Diagn. Invest. 18:18-28.

Galiza G.J.N., Silva M.L.C.R., Dantas A.F.M., Simões S.V.D. \& Riet-Correa F. 2010. Doenças do sistema nervoso de bovinos no semiárido nordestino. Pesq. Vet. Bras. 30(3):267-276.

Ginn P.E., Mansell J.E.K.L. \& Rakich P.M. 2007. Skin and appendages, p.553781. In: Maxie M.G. (Ed.), Jubb, Kennedy \& Palmer's Pathology of Domestic Animals. Vol.1. 5th ed. Elsevier, Philadelphia. 899p.

Gomes L.I., Rocha M.A., Costa E.A., Lobato Z.I.P., Mendes L.C.N., Borges A.S., Leite R.C. \& Barbosa-Stancioli E.F. 2002. Detecção de herpesvírus bovino-5 (BoHV-5) em bovinos do sudeste brasileiro. Arq. Bras. Med. Vet. 54(2):217-220.

IBGE 2012. Produção da Pecuária Municipal (PPM). Instituto Brasileiro de Geografia e Estatística, Rio de Janeiro, RJ. 63p.

IMEA 2014. 3ํ Levantamento das Intenções de Confinamento em 2014. Instituto Matogrossense de Economia Agropecuária. 4p.

INDEA 2014. Instituto de Defesa Agropecuária do Estado de Mato Grosso. Disponível em <http://www.indea.mt.gov.br/defesa-sanitaria/downloads/relatorio-de-populacao-bovina-existente-novembro2014/134647> Acesso em 12 fev. 2015.

Johnson R. \& Kaneene J.B. 1992. Bovine leukemia vírus and enzootic bovine leukosis. Vet. Bull. 62:287-312.

Lemos A.A.L. \& Nakazato L. 2007. Carcinomas de base de chifre, p.656658. In: Riet-Correa F., Schild A.L., Lemos R.A.A. \& Borges J.R.J. (Eds), Doenças de Ruminantes e Equídeos. 3aㅡ ed. Pallotti, São Paulo.

Lucena R.B., Pierezan F., Kommers G.D., Irigoyen L.F., Fighera R.A. \& Barros C.S.L. 2010. Doenças de bovinos no Sul do Brasil: 6.706 casos. Pesq. Vet. Bras. 30(5):428-434.

MacLachlan N.J. \& Kennedy P.C. 2002. Tumors of genital systems, p.547573. In: Meuten D.J. (Ed.). Tumors in Domestic Animals. 4th ed. Iowa State Press, Ames. 788p.

Madruga C.R., Aycardi E., Kesller R.H., Schenk M.A.M., Figueiredo G.R. \& Curvo J.B.E. 1984. Níveis de anticorpos anti-Babesia bigemina e Babesia bovis em bezerros da raça Nelore, Ibagé e cruzamentos de Nelore. Pesq. Agropec. Bras. 19:1163-1168.

Madruga C.R., Berne M.E.A., Kessler R.H., Gomes R.F.C., Lima J.G. \& Schenk M.A.M. 1986. Diagnóstico da tristeza parasitária bovina no Estado do Mato Grosso do Sul: inquérito de opinião. Circ. Téc. 18, Embrapa-CNPGC, Campo Grande. 32p.

MAPA 2009. Controle da Raiva dos Herbívoros: manual técnico. Secretaria de Defesa Agropecuária, Ministério da Agricultura, Pecuária e Abastecimento, Brasília. 124p. Disponível em <http://www.agricultura.gov. $\mathrm{br} /$ arq_editor/file/Aniamal/programa\%20nacional\%20dos\%20herbivoros/manual $\% 20$ tecnico $\% 20$ para $\% 20$ controle $\% 20$ da $\% 20$ raiva.pdf> Acesso em 5 mai. 2015.

Marcolongo-Pereira C., Sallis E.S.V., Grecco F.B., Raffi M.B., Soares M.P. \& Schild A.L. 2011. Raiva em bovinos na Região Sul do Rio Grande do Sul: epidemiologia e diagnóstico imunohistoquímico. Pesq. Vet. Bras. 31(4):331-335.

Martin S.W., Meek A.H., Davis D.G., Thonsom R.G., Johnson J.A., Lopez A., Stephens L., Curtis R.A., Prescott J.F., Rosendal S., Savan M., Zubaidy A.J. \& Bolton M.R. 1980. Factors associated with mortality in feedlot cattle: the Bruce County Beef Cattle Project. Can. J. Comp. Med. 44:1-10.

Martin S.W., Meek A.H., Davis D.G., Johnson J.A. \& Curtis R.A. 1981. Factors associated with morbidity and mortality in feedlot calves: the Bruce County Beef Project, year two. Can. J. Comp. Med. 45:103-112.

Mendonça F.S., Dória R.G.S., Schein F.B., Freitas S.H., Nakazato L., Boabaid F.M., Paula D.A.J., Dutra V. \& Colodel E.M. 2008. Febre catarral maligna em bovinos no Estado de Mato Grosso. Pesq. Vet. Bras. 28(3):155-160.

Mori A.E. \& Lemos R.A.A. 1998. Raiva, p.47-58 In: Lemos R.A.A. (Ed.), Prin- 
cipais Enfermidades de Bovinos de Corte do Mato Grosso do Sul: reconhecimento e diagnóstico. Universidade Federal de Mato Grosso do Sul, Campo Grande, MS.

Pedroso P.M.O., Colodel E.M., Pescador C.A., Arruda L.P. \& Driemeier D. 2009. Aspectos clínicos e patológicos em bovinos afetados por raiva com especial referência ao mapeamento do antígeno rábico por imunohistoquímica. Pesq. Vet. Bras. 29(11):899-904.

Pessoa C.R.M., Medeiros R.M.T. \& Riet-Correa F. 2013. Importância econômica, epidemiologia e controle das intoxicações por plantas no Brasil. Pesq. Vet. Bras. 33(6):752-758.

Rech R.R., Schild A.L., Driemeier D., Garmatz S.L., Oliveira F.N., Riet-Correa F. \& Barros C.S.L. 2005. Febre catarral maligna em bovinos no Rio Grande do Sul. Pesq. Vet. Bras. 25(2):97-105.

Ribas N.L.K.S., Carvalho R.I., Santos A.C., Valençoela R.A., Gouveia A.F., Castro M.B., Mori A.E. \& Lemos R.A.A. 2013. Doenças do sistema nervoso de bovinos no Mato Grosso do Sul: 1082 casos. Pesq. Vet. Bras. 33(10):1183-1194.

Ribeiro M., Damasceno E.S., Teza J., Bertipaglia K.S.S., Schenkel D.M., Silva L.A., Ducatti K.R. \& Furlan F.H. 2014. Prevalência de carcinoma escamoso periocular em bovinos na região norte de Mato Grosso. Disponível em <http://www2.ufrb.edu.br/apa/documentos/category/9-neoplasias? download=143:7036> Acesso em 25 jul. 2015.

Riet-Correa F., Vidor T., Schild A.L. \& Méndez M.C. 1989. Meningoencefalite e necrose do córtex cerebral em bovinos causadas por Herpes Vírus Bovino-1. Pesq. Vet. Bras. 9:13-16.

Riet-Correa F. \& Schild A.L. 1995. Doenças diagnosticadas pelo Laboratório Regional de Diagnóstico no ano de 1994 e comentários sobre algumas doenças. Bolm Lab. Reg. Diagn., UFPel, n.15, Pelotas, RS. 46p.

Rodrigues W.B., Otonel R.A.A., Fritzen J.T.T., Gardinali N.R., Colodel E.M., Alfieri A.F. \& Alfieri A.A. 2011. Natural infection of calf with an atypical bovine pestivirus (BVDV-3). XXII Encontro Nacional de Virologia, VI Encontro de Virologia do Mercosul, Atibaia. Virus Reviews and Research 16:74-74.

Sanches A.W.D., Langohr I.M., Stigger A.L. \& Barros C.S.L. 2000. Doenças do sistema nervoso central em bovinos no Sul do Brasil. Pesq. Vet. Bras. 20(3):113-118.

Sant'Ana F.J.F., Rissi D.R., Lucena R.B., Lemos R.A.A., Nogueira A.P. \& Barros C.S.L. 2009a. Polioencefalomalacia em bovinos: epidemiologia, sinais clínicos e distribuição das lesões no encéfalo. Pesq. Vet. Bras. 29(7):487-497.

Sant'Ana F.J.F., Lemos R.A.A., Nogueira A.P.A., Togni M., Tessele B. \& Barros C.S.L. 2009b. Polioencefalomalacia em ruminantes. Pesq. Vet. Bras. 29(9):681-694.

Santos B.S., Pinto A.P., Aniz A.C.M., Almeida A.P.M.G., Franco G.L., Guimarães E.B. \& Lemos R.A.A. 2012. Mortalidade de bovinos zebuínos por hipotermia em Mato Grosso do Sul. Pesq. Vet. Bras. 32(3):204-210.
Silva R.A., Silva N.M. \& Meneses P.R.V. 1974. Ocorrência do vírus da raiva na medula e bulbo de equinos na doença natural e sua ausência nas diferentes regiões do sistema nervoso central e outros tecidos. Pesq. Agropec. Bras., Sér. Vet. 9:29-31.

Silveira S., Weber M.N., Mósena A.C.S., Silva M.S., Streck A.F., Pescador C.A., Flores E.F., Weiblen R., Driemeier D., Ridpath J.F. \& Canal C.W. 2015. Genetic diversity of Brazilian bovine pestiviruses detected between 1995 and 2014. Transboundary and Emerging Diseases. Doi: 10.1111/ tbed.12427

Tokarnia C.H., Brito F.M., Barbosa J.D., Peixoto P.V. \& Döbereiner J. 2012. Plantas Tóxicas do Brasil. 2 $2^{\mathrm{a}}$ ed. Helianthus, Rio de Janeiro. 586p.

Ubiali D.G., Caldeira F.H.B., Moraes L.G., Neto W.S.P., Antoniassi N.A.B., Arruda L.P., Souza M.A. \& Colodel E.M. 2008. Causas de enfermidades com sinais clínicos nervosos em bovinos no Estado de Mato Grosso entre janeiro de 2005 e abril de 2008 diagnosticadas no LPV-UFMT. Anais VI Encontro Nacional de Diagnóstico Veterinário (Endivet), Campo Grande, MS.

Ubiali D.G., Cruz R.A.S., Lana M.V.C., Meireles Y.S., Néspoli P.B., Souza M.A., Colodel E.M. \& Pescador C.A. 2011. Spinal cord compression in cattle after the use an oily vacccine. Pesq. Vet. Bras. 31(11):997-999.

Ubiali D.G., Silva R.G.F., Oliveira L.P., Moraes L.G., Caldeira F.H.B., Pescador C.A. \& Colodel E.M. 2013. Obstrução intestinal em bovinos causada pelo consumo de Stylosanthes ssp. (Fabaceae Papilionoideae). Pesq. Vet. Bras. 33(2):148-154.

USDA 2014. Foreign Agricultural Service. United States Department of Agriculture, Washington, DC. Disponível em <http://apps.fas.usda. gov/ psd online/> Acesso 12 jan. 2015.

Waldner L.C., Kennedy R.I., Rosengren L. \& Clark E.G. 2009. A field study of culling and mortality in beef cows of western Canada. Can. J. Vet. Res. 50:491-499.

Watson E.N., David G.P. \& Cook A.J.C. 2008. Review of diagnostic laboratory submissions of adult cattle 'found dead' in England and Wales in 2004. Vet. Rec. 163:531-535.

Weber M.N., Mósena A.C.S., Simões S.V.D., Almeida L.L., Pessoa C.R.M., Budaszewski R.F., Silva T.R., Ridpath J.F., Riet-Correa F., Driemeier D. \& Canal C.W. 2014. Clinical presentation resembling mucosal disease associated with 'HoBi'-like pestivirus in a field outbreak. Transboundary and Emerging Diseases. Doi: 10.1111/tbed.12223

Wells G.A.H., Scott A.C., Johnson C.R., Gunning R.F., Hancock RD., Jeffrey M., Dawson M. \& Bradley R. 1987. A novel progressive spongiform encephalopathy in cattle. Vet. Rec. 121:419-420.

Wettimuny S.G.S. 1974. Neoplastic and proliferative lesions of the vulva of Ayrshire cattle in Sri Lanka. Ceylon Med. J. 22(1/2):17-20.

Will R.G., Ironside J.W., Zeidler M., Cousens S.N., Estibeiro K., Alpeovitch A., Poser S., Pocchiari M., Hofman A. \& Smith P.G. 1996. A new variant of Creutzfeldt-Jakob disease in the UK. Lancet 347:921-925. 